\title{
Childhood diet and asthma and atopy at 8 years of age: the PIAMA birth cohort study
}

\author{
S.M. Willers*, A.H. Wijga* , B. Brunekreef*", S. Scholtens ${ }^{+}$, D.S. Postma ${ }^{\S}$, \\ M. Kerkhof ${ }^{+}$, J.C. de Jongste ${ }^{f}$ and H.A. Smit
}

ABSTRACT: Diet may affect the development of asthma. We investigated whether asthma or atopy outcomes at $\mathbf{8}$ yrs of age were associated with long-term dietary exposure, and whether associations were different for consumption at early or later age.

The Prevention and Incidence of Asthma and Mite Allergy (PIAMA) birth cohort enrolled 4,146 participants at baseline, who were followed up to 8 yrs of age. Dietary intakes of interest were fruit, vegetables, brown/wholemeal bread, fish, milk, butter and margarine. Associations between food intake at early (2-3 yrs) and later (7-8 yrs) age, and long-term intake, asthma and atopy at 8 yrs of age were calculated by logistic regression.

Complete longitudinal dietary data for at least one of the food groups were available for 2,870 children. Fruit consumption at early age was associated with reduced asthma symptoms (OR per 1 consumption day per week increase $0.93,95 \% \mathrm{Cl} 0.85-1.00)$. Long-term fruit intake was inversely associated with asthma symptoms (OR $0.90,95 \% \mathrm{Cl} 0.82-0.99$ ) and sensitisation to inhaled allergens (OR $0.90,95 \% \mathrm{Cl} 0.82-0.99$ ). We found no consistent associations between diet and outcomes for other foods.

This study indicates no consistent effects of increased early or late consumption, or long-term intake of certain foods on asthma and atopy in 8-yr-olds, with a possible exception for fruit.

KEYWORDS: Asthma, atopy, children, diet

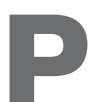

rosperity-associated changes in diet, and more specifically the reduced consumption of antioxidant rich foods and change in dietary fat intake, have been linked to the increase in asthma and allergic disease in the last few decades [1, 2]. Previous studies have reported beneficial associations between a higher consumption of fruit [3-5], vegetables [4], fish [5, 6], full-fat dairy products [7] and wholegrain products [7, 8], and symptoms of asthma or allergy in children, while harmful associations have been reported for margarine and salt intake [9, 10]. It has been hypothesised that dietary exposure in early life (from conception to 2 yrs of age) might be particularly important in the development of childhood asthma, because the airways and immune system are developing during this period [11]. Most of the previous studies that assessed the effect of diet on asthma in children related dietary data that was obtained at one point in time to asthma and/or allergy outcomes at the same point in time or a (few) year(s) later. Results of these studies do not give information about the effects of long-term dietary exposure or differences in effects of dietary habits at early or later age. The use of longitudinal dietary data would give more insight in these effects, which may be crucial for (the timing of) dietary preventive strategies for childhood asthma and allergy.

The Prevention and Incidence of Asthma and Mite Allergy (PIAMA) birth cohort study has annual follow-up dietary data from birth to 8 yrs of age. The aim of the present study was to investigate the effects of long-term dietary exposure from 2 to 8 yrs of age, and dietary habits at early and later age on symptoms and clinical outcomes of asthma and atopy at 8 yrs of age.

\section{METHODS}

\section{Study population and study design}

In 1996, a large birth cohort study was set up to investigate the Prevention and Incidence of

\section{AFFILIATIONS}

*Institute for Risk Assessment Sciences (IRAS), Division Environmental Epidemiology, Utrecht University,

- Julius Centre for Health Sciences and Primary Care, University Medical Centre Utrecht, Utrecht,

${ }^{\#}$ Centre for Prevention and Health Services Research, National Institute for Public Health and the Environment (RIVM), Bilthoven, ${ }^{+}$Dept of Epidemiology, University Medical Center Groningen,

${ }^{\S}$ Dept of Pulmonology, Beatrix Children's Hospital, University Medical Center Groningen,

Groningen, and

${ }^{f}$ Dept of Paediatrics/Respiratory Medicine, Erasmus Medical Centre/ Sophia Children's Hospital, Rotterdam, The Netherlands.

CORRESPONDENCE

A.H. Wijga

Centre for Prevention and Health Services Research

National Institute for Public Health and the Environment (RIVM) PO Box 1

3720 BA Bilthoven

The Netherlands

E-mail: alet.wijga@rivm.nl

Received:

July 072009

Accepted after revision:

Oct 272010

First published online:

Nov 252010 
Asthma and Mite Allergy (PIAMA). Details of the design of the PIAMA study have been published previously [12]. Briefly, 10,232 pregnant females were screened for atopy while visiting their pre-natal health clinic [13]. Based on this screening, 2,779 atopic and 5,083 nonatopic females were invited to participate in the study, of whom 1,327 atopic and 2,819 nonatopic females agreed. In the intervention part of PIAMA, which was designed to study the effect of mite allergen avoidance by means of mite-impermeable mattress and pillow covers, only children born to atopic mothers ("high-risk" children) were enrolled. In the observational natural history part, children of atopic and those of nonatopic mothers ("low-risk" children) were enrolled. $183(\sim 4.5 \%)$ mothers were lost to follow-up before any data on the child had been collected, so 3,963 children continued in the study. A flow chart of the recruitment and response of the study population is shown in online supplementary figure E1.

Questionnaires contained the International Study of Asthma and Allergies in Childhood (ISAAC) core questions on asthma, rhinitis and eczema [14], as well as various questions on lifestyle factors (nutrition, pets, home characteristics, etc.), and were sent to the participants at 3 months of age, and annually from 1 to 8 yrs of age. The Medical Ethical Committees of the participating institutes approved the study and all participants gave written informed consent.

\section{Assessment of the child's diet from 2 to 8 yrs of age}

Longitudinal data on the child's diet were derived from the annual questionnaires from 2 to $8 \mathrm{yrs}$ of age. These questionnaires enquired about the frequency of consumption of approximately 30-35 different foods or food groups in the preceding month. The foods and food groups of interest were the ones high in antioxidants (fruit, vegetables and brown/ wholemeal bread), n-3 fatty acids (fish), n-6 fatty acids (margarine) and milk fat (full-cream milk, semi-skimmed milk and butter). Answer options for the frequency of consumption were: 1) never; 2) less than once a week; 3) $1-2$ days week $^{-1}$; 4) 3-5 days.week ${ }^{-1}$; and 5) 6-7 days.week ${ }^{-1}$. These answer options were translated into continuous variables in consumption days per week: option $1=0$; option $2=0.5$; option $3=1.5$; option $4=4$; and option $5=6.5$ consumption days per week. The original data of the frequency of consumption of the different foods and food groups from 2 to 8 yrs of age is presented in online supplementary tables E1a-c.

\section{Definition of early and late consumption, and long-term intake}

Consumption at early age was defined as the mean consumption frequency (in consumption days per week) of the questionnaires at both 2 and 3 yrs of age, while consumption at later age was defined as the mean consumption frequency of the questionnaires at both 7 and 8 yrs of age.

Long-term intake was defined as the mean consumption frequency (in consumption days per week) of all questionnaires from 2 to 8 yrs of age.

\section{Children's health outcomes}

The child's health outcomes of interest were wheeze, dyspnoea and inhaled steroid use at 8 yrs of age derived from the questionnaire, sensitisation against inhalant and food allergens, and bronchial hyperresponsiveness (BHR) at 8 yrs of age assessed by medical examination. Besides these specific health outcomes, a composite variable "asthma symptoms" was used within the PIAMA study. A child was defined as having "asthma symptoms" when the parents reported one or more attacks of wheeze, and/or one or more events of dyspnoea and/ or prescription of inhaled steroids for respiratory problems in the previous 12 months. A child who had none of these characteristics was defined as not having "asthma symptoms". This composite variable "asthma symptoms" was designed to include children with prevalent wheeze or dyspnoea symptoms, but also children who might not have had these symptoms because they used asthma medication (inhaled steroids) during the previous 12 months. In this way, symptomatic children could be compared with a "clean" reference group of children that had neither wheeze or dyspnoea, nor used inhaled steroids for respiratory problems.

At 8 yrs of age, children from the intervention part, the "highrisk" natural history part and a random sample of the "lowrisk" natural history part, drawn at the beginning of the PIAMA study, were invited for hospital-based medical examination. Participants who were not able to come to the hospital clinic and the remaining group of "low-risk" natural history children were invited for a community-based medical examination either at a local community health centre or at home. Both the hospitaland community-based medical examinations included blood sampling for the assessment of total and specific immunoglobulin (Ig)E levels. Children were considered to be sensitised against inhalant allergens if one or more allergen-specific $\operatorname{IgE}$ level to house dust mite (Dermatophagoides pteronyssinus), cat, dog, birch (Betula verrucosa), grass (Dactylis glomerata) or fungus (Alternaria alternata) were $\geqslant 0.35 \mathrm{IU} \cdot \mathrm{mL}^{-1}$. Sensitisation to food allergens was defined as a high level of allergen-specific IgE to milk or egg (also $\geqslant 0.35 \mathrm{IU} \cdot \mathrm{mL}^{-1}$ ). A methacholine provocation test as indicator for BHR was only conducted within the hospital-based medical examination. Children were defined as having BHR when the cumulative dose of methacholine bromide causing a $20 \%$ decrease in forced expiratory volume in $1 \mathrm{~s}$ was $\leqslant 0.61 \mathrm{mg}$.

\section{Statistical analyses}

Univariate and multivariate logistic regression analyses were used to assess associations between 1 consumption day per week increase in consumption of the different foods and food groups during childhood, and asthma and allergy outcomes at 8 yrs of age. This was done for average consumption at early age ( 2 and 3 yrs of age), later age ( 7 and 8 yrs of age) and average long-term intake from 2 to 8 yrs of age. Given the often skewed distribution of the food consumption categories, an analysis by tertiles or quartiles was not usually possible, so a more continuous representation of exposure gave more easily interpretable results, similarly for all investigated foods and food groups.

The analyses for consumption at early age were performed on children with the respective food intake data at both 2 and $3 \mathrm{yrs}$ of age, as well as the respective outcome at $8 \mathrm{yrs}$ of age and all confounders. For consumption at later age, food data was present at both 7 and 8 yrs of age, while for long-term consumption, food data was present at all ages from 2 to 8 yrs. 
TABLE 1 Characteristics of the study population at baseline and complete cases at 8 yrs of age

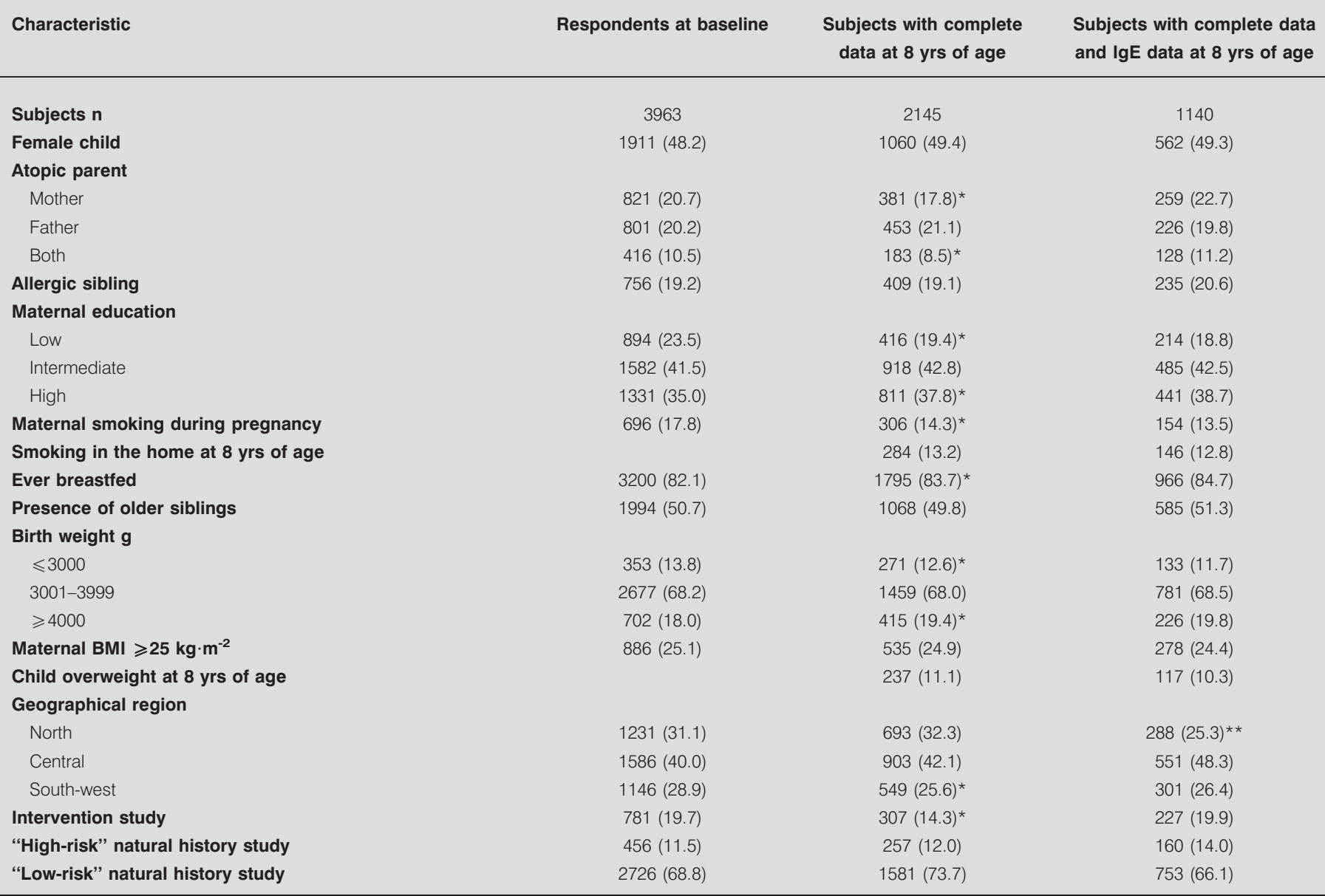

Data are presented as $n(\%)$, unless otherwise stated. Ig: immunoglobulin; BMl: body mass index. *: $p<0.05$ for complete cases included in the analyses compared with noncomplete cases; **: $\mathrm{p}<0.05$ for complete cases with IgE data are compared with complete cases without lgE data.

Covariates included as potential confounding factors in the multivariate model were: sex; parental atopy; maternal education (low, intermediate or high); maternal smoking during pregnancy; smoking in the home by the mother, father or others at 8 yrs of age; breast feeding (yes or no); presence of older siblings; birth weight $(\leqslant 3,000,3,001-3999$ and $\geqslant 4,000 \mathrm{~g})$;

TABLE 2 Mean and median early, late and long-term food consumption in days per week

\begin{tabular}{|c|c|c|c|c|c|c|c|c|c|}
\hline & \multicolumn{3}{|c|}{ Early consumption } & \multicolumn{3}{|c|}{ Late consumption } & \multicolumn{3}{|c|}{ Long-term intake } \\
\hline & Subjects $\mathbf{n}$ & Mean $\pm S D$ & Median (IQR) & Subjects $\mathrm{n}$ & Mean $\pm S D$ & Median (IQR) & Subjects n & Mean $\pm S D$ & Median (IQR) \\
\hline Fresh fruit & 3574 & $5.3 \pm 1.6$ & $6.5(2.5)$ & 3088 & $5.1 \pm 1.7$ & $5.3(2.5)$ & 2815 & $5.3 \pm 1.4$ & $5.8(2.1)$ \\
\hline Cooked vegetables & 3571 & $5.0 \pm 1.5$ & $5.3(2.5)$ & 3094 & $4.7 \pm 1.4$ & $4.0(2.5)$ & 2793 & $4.8 \pm 1.2$ & $4.7(1.8)$ \\
\hline $\begin{array}{l}\text { Brown/wholemeal } \\
\text { bread }\end{array}$ & 3584 & $5.9 \pm 1.3$ & $6.5(0.0)$ & 3107 & $5.8 \pm 1.5$ & $6.5(1.3)$ & 2839 & $5.9 \pm 1.2$ & $6.5(0.7)$ \\
\hline Fish & 3540 & $0.8 \pm 0.7$ & $0.5(0.5)$ & 3073 & $0.9 \pm 0.7$ & $0.5(1.0)$ & 2716 & $0.8 \pm 0.6$ & $0.7(0.6)$ \\
\hline Full-cream milk & 3545 & $1.9 \pm 2.5$ & $0.0(3.3)$ & 3086 & $0.4 \pm 1.4$ & $0.0(0.0)$ & 2756 & $1.0 \pm 1.6$ & $0.1(0.7)$ \\
\hline Semi-skimmed milk & 3565 & $3.9 \pm 2.6$ & $4.0(5.8)$ & 3089 & $4.1 \pm 2.7$ & $5.3(5.5)$ & 2778 & $4.1 \pm 2.2$ & $4.7(3.9)$ \\
\hline Butter & 3567 & $0.6 \pm 1.6$ & $0.0(0.3)$ & 3088 & $0.5 \pm 1.4$ & $0.0(0.3)$ & 2818 & $0.6 \pm 1.3$ & $0.1(0.4)$ \\
\hline Margarine & 3556 & $5.1 \pm 2.0$ & $6.5(2.5)$ & 3082 & $5.2 \pm 2.2$ & $6.5(2.5)$ & 2794 & $5.3 \pm 1.7$ & $6.1(1.6)$ \\
\hline
\end{tabular}

IQR: interquartile range. 
overweight of the mother (maternal body mass index (BMI) $\geqslant 25 \mathrm{~kg} \cdot \mathrm{m}^{-2}$ assessed at 1-yr follow-up); overweight of the child at 8 yrs of age (BMI was calculated, and overweight was defined according to age- and sex-specific international standards that use cut-off points equivalent to the $25 \mathrm{~kg} \cdot \mathrm{m}^{-2}$ cut-off that is commonly used for adults [15]); geographical region (north, central or south-west); and study arm (intervention study, and "high-risk" or "low-risk" natural history study). All analyses were carried out using SAS for Windows version 9.1 (SAS Institute, Cary, NC, USA).

\section{RESULTS}

At 8-yr follow-up, questionnaire information was obtained for 3,269 children. 1,554 participants were invited for the hospitalbased medical examination while 1,964 participants were invited for the community-based medical examination, of whom 1,133 and 1,081 children participated, respectively. The methacholine provocation test, which was included in the hospital-based examination, was successful for 938 children. IgE levels in blood samples were analysed for 1,713 children. Complete dietary data from 2 to 8 yrs of age for at least one of the investigated food groups were obtained for 2,870 children.

The characteristics of respondents at baseline compared to complete cases at 8 yrs of age (complete dietary data from 2 to 8 yrs of age for at least one of the investigated food groups, 8yr questionnaire and all confounders) and complete cases with $\operatorname{IgE}$ data at 8 yrs of age are shown in table 1.

Children with complete data at 8 yrs of age were less likely to have an atopic mother or a mother who smoked during pregnancy, or to be from south-west region compared with the children at baseline. Furthermore, these children were more likely to have a mother with a higher educational level and to have a higher mean birth weight compared with the children at baseline. Children with $\operatorname{IgE}$ data at 8 yrs of age were less likely to be from northern region. However, the magnitude of the differences between populations was small.

Table 2 shows the mean and median consumption of the investigated foods and food groups in consumption days per week.

\begin{tabular}{|c|c|c|c|}
\hline TABLE 3 & \multicolumn{3}{|c|}{$\begin{array}{l}\text { Prevalence of health outcome variables at } 8 \text { yrs } \\
\text { of age }\end{array}$} \\
\hline Health outc & & $\mathbf{N}^{\#}$ & n (\%) \\
\hline \multicolumn{2}{|c|}{ Wheeze in the previous 12 months } & 3269 & $213(6.5)$ \\
\hline \multicolumn{2}{|c|}{ Dyspnoea in the previous 12 months } & 3269 & $293(9.0)$ \\
\hline \multicolumn{2}{|c|}{$\begin{array}{l}\text { Use of inhaled steroids in the previous } \\
12 \text { months }\end{array}$} & 3269 & $208(6.4)$ \\
\hline \multicolumn{2}{|c|}{ Asthma symptoms $\pi$} & 3269 & $425(13.0)$ \\
\hline \multicolumn{2}{|c|}{ Sensitisation to inhaled allergens ${ }^{+}$} & 1713 & $550(32.1)$ \\
\hline \multicolumn{2}{|c|}{ Sensitisation to food allergens $s^{5}$} & 1713 & $285(16.6)$ \\
\hline \multicolumn{2}{|l|}{ BHR } & 938 & $402(42.9)$ \\
\hline \multicolumn{4}{|c|}{$\begin{array}{l}\text { BHR: bronchial hyperresponsiveness. }{ }^{\#}: \text { number of children with questionnaire } \\
\text { information, immunoglobulin } \mathrm{E} \text { data or methacholine provocation test con- } \\
\text { ducted. ": composite variable of wheeze, dyspnoea or inhaled steroid use; } \\
+ \text { + house dust mite, cat. dog. grass, birch or funqus: }{ }^{\text {s: }} \text { milk or egg. }\end{array}$} \\
\hline
\end{tabular}

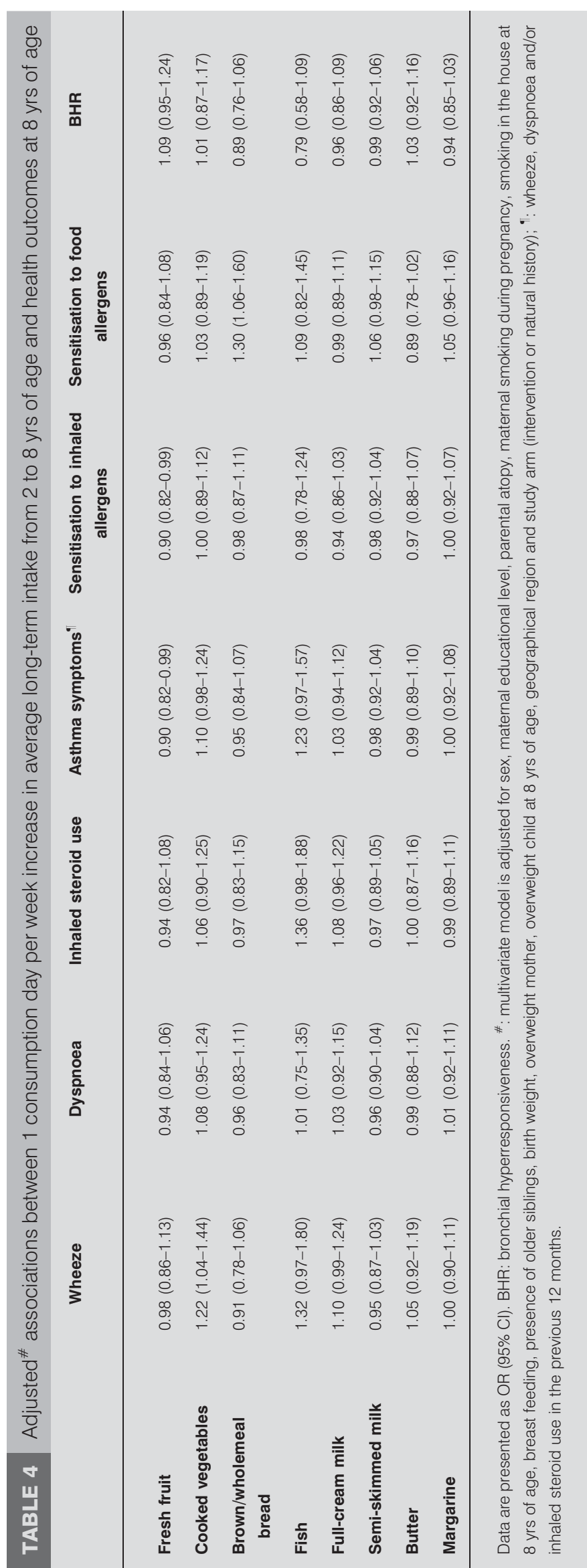




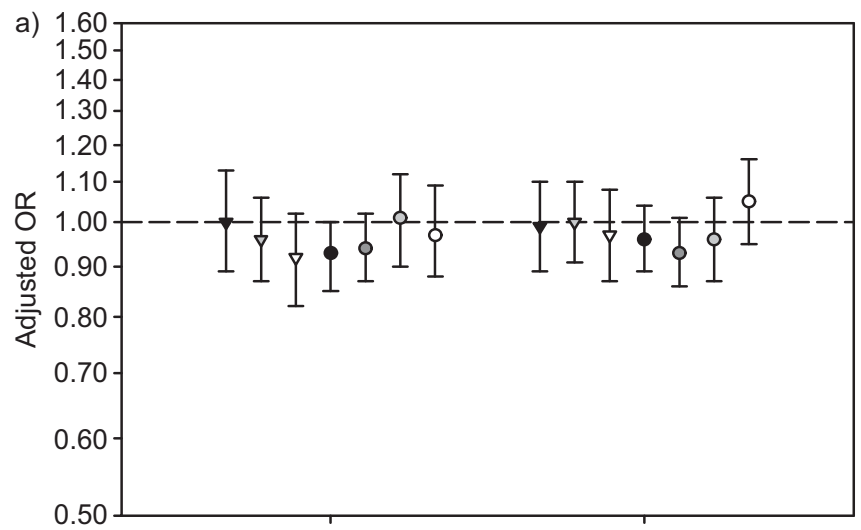

b)
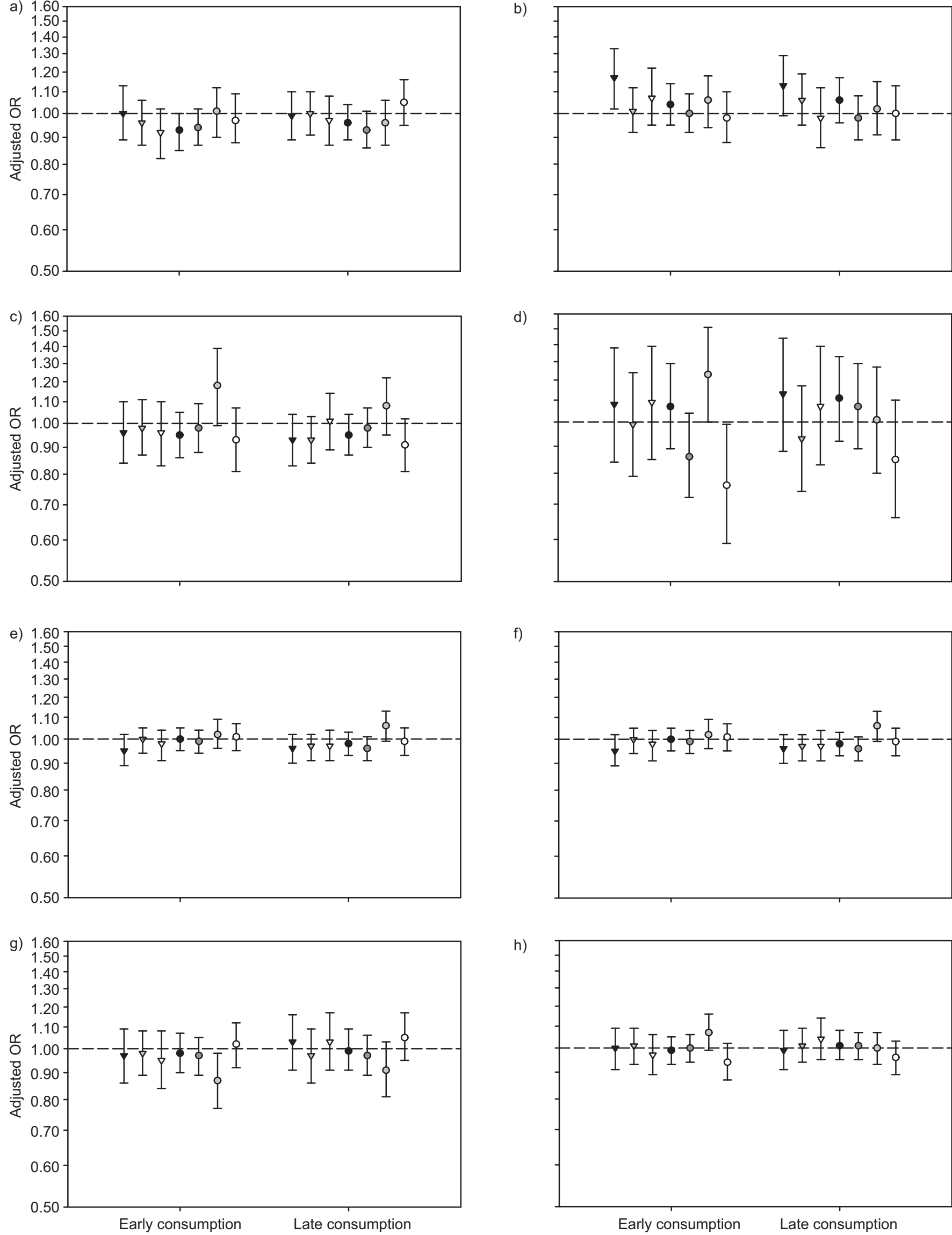

d)

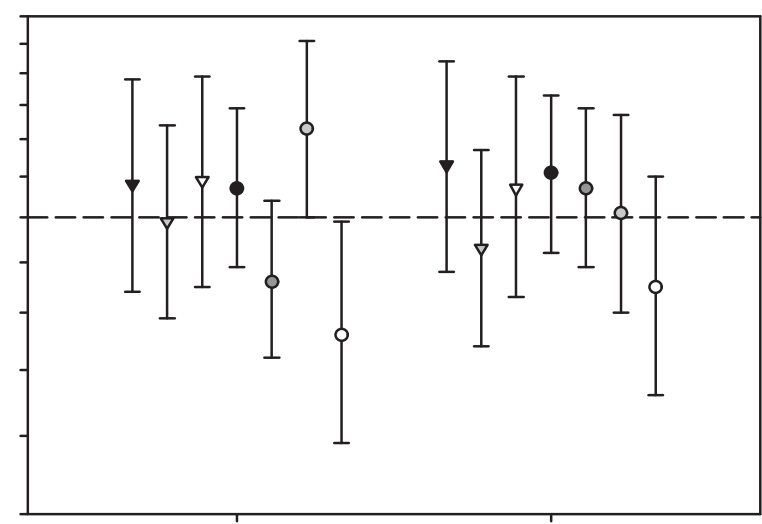

f)

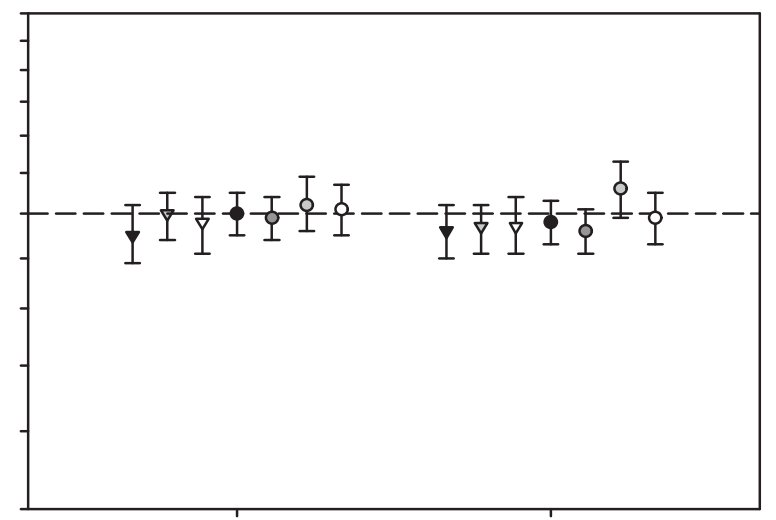

h)

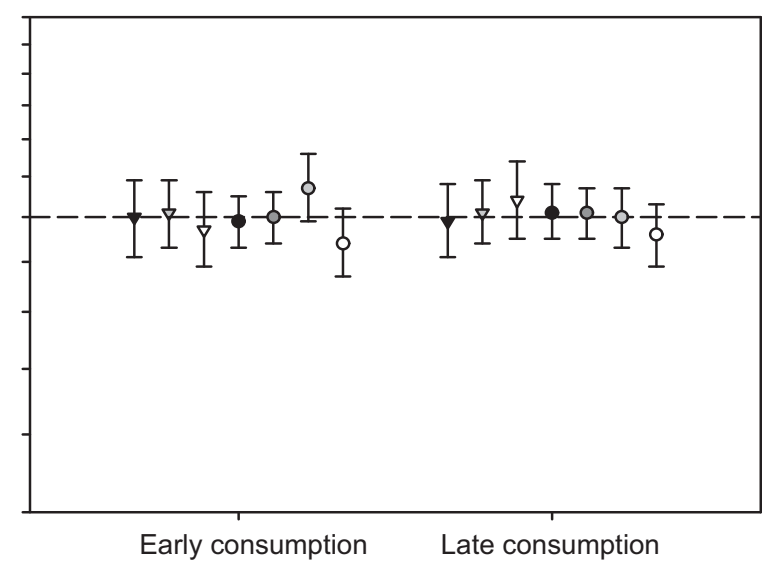




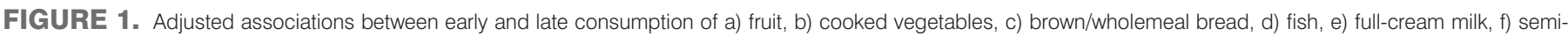

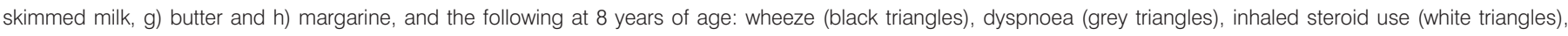

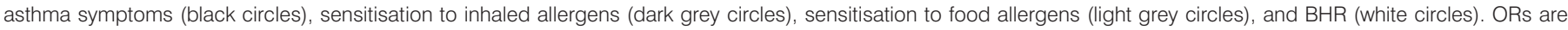

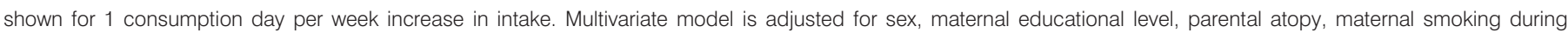

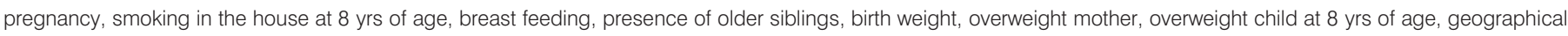
region and study arm (intervention or natural history). Whiskers represent $95 \% \mathrm{Cl}$.

At early age, most children consumed fruit, brown/wholemeal bread and margarine daily. Cooked vegetables and semiskimmed milk were generally consumed on 5 and 4 day$\mathrm{s} \cdot$ week $^{-1}$, respectively, while fish, full-cream milk and butter were generally consumed less than once a week. At later age, consumption of fish, semi-skimmed milk and margarine increased, while consumption of fruit, vegetables and fullcream milk decreased. Average long-term intake was highest for brown/wholemeal bread, fruit and margarine, while it was lowest for butter and fish.

Prevalence of health outcomes at 8 yrs of age are shown in table 3.

Among the 8-yr-olds with questionnaire data, dyspnoea was the most commonly reported symptom $(9.0 \%)$. Of the children with IgE data, $32.1 \%$ were sensitised to inhaled allergens, while $16.6 \%$ were sensitised to milk or egg. The methacholine provocation test was positive in $42.9 \%$ of the children. However, the subgroup of children that performed a methacholine provocation test contained a larger proportion of "high-risk" children than the total study population.

Adjusted associations between average long-term intake of the different foods and food groups from 2 to $8 \mathrm{yrs}$ of age and the different asthma and atopy outcomes at $8 \mathrm{yrs}$ of age are shown in table 4 . There were no consistent associations between increased long-term intake of fish, fullcream milk, semi-skimmed milk, butter or margarine on symptoms of asthma, atopy or BHR at 8 yrs of age. Longterm intake of fresh fruit was significantly inversely associated with asthma symptoms (OR per 1 consumption day per week increase $0.90,95 \%$ CI $0.82-0.99)$ and sensitisation to inhalant allergens (OR 0.90, 95\% CI 0.82-0.99). Longterm intake of cooked vegetables and brown/wholemeal bread consumption were significantly positively associated with wheeze and sensitisation to food allergens (OR 1.22 (95\% CI 1.04-1.44) and OR 1.30 (95\% CI 1.06-1.60), respectively). The effects of inclusion of potential confounding factors in the different models were minimal. After additionally adjusting for sensitisation to pollen or hay fever at 8 yrs of age, the association between long-term fruit intake and asthma symptoms became borderline significant (OR 0.92, 95\% CI 0.83-1.01).

Figure 1 shows the adjusted associations between 1 consumption day increase in the investigated foods and food groups at early age, and later age and symptoms of asthma, atopy and BHR at 8 yrs of age.

There were no associations between increased consumption of brown/wholemeal bread, semi-skimmed milk or margarine at either early or later age on symptoms of asthma, atopy or BHR. The results did show an inverse association between increased fruit consumption at early age and asthma symptoms (OR 0.93, $95 \%$ CI 0.85-1.00). Increased fish consumption at early age was significantly inversely associated with BHR (OR 0.76, 95\% CI 0.59-0.99) and borderline significantly positively associated with sensitisation to food allergens at 8 yrs of age (OR 1.23, 95\% CI 1.00-1.51). Furthermore, we found significantly positive associations between increased consumption of cooked vegetables at early age and full cream milk at later age and wheeze at 8 yrs of age (OR 1.17 (95\% CI 1.02-1.33) and OR 1.14 (95\% CI 1.02-1.27), respectively) and a significantly inverse association between increased consumption of butter at early age and sensitisation to food allergens at 8 yrs of age (OR $0.87,95 \%$ CI $0.77-0.98)$.

\section{DISCUSSION}

We have investigated associations between long-term dietary intake, and dietary intake in early and later childhood with symptoms and clinical outcomes of asthma and atopy at $8 \mathrm{yrs}$ of age. No consistent associations were found for long-term dietary consumption or consumption at early or later age on outcomes at 8 yrs of age, except for fruit. Increased fruit consumption at early age was beneficially associated with inhaled steroid use, asthma symptoms and sensitisation to inhaled allergens; however, these associations were only borderline statistically significant. Increased long-term fruit intake was significantly inversely associated with asthma symptoms and sensitization to inhalant allergens.

The associations between fruit consumption and childhood asthma and atopy we observed in this study were not very strong. Results from previous studies in children are ambiguous as well. Some studies have found beneficial associations between fruit consumption and lung function [16], wheeze [17], cough [4, 5] or rhinitis [18], whereas other studies did not find associations between fruit consumption and lung function [19], asthma or wheeze [7, 20-22], or only found associations with specific types of fruit, especially thoes high in vitamin $C$ $[3,8,23]$. High fruit consumption probably leads to a higher antioxidant status in the body, which provides more protection against oxidative damage through infections, passive smoking and air pollution [24, 25].

The inverse relationships between increased fruit consumption, and asthma symptoms and sensitisation to inhaled allergens are potentially attributable to allergen cross-reactivity between pollen and specific types of fruit [26]. This would lead to reverse causation: asthmatic children allergic to pollen might be more likely to avoid certain fruits due to food allergic reactions. However, additional adjustment of the analyses for hay fever and (in those with sensitisation data) for pollen sensitisation did not substantially change the results.

Maintenance of high fruit consumption over a longer period of time may reflect a more healthy diet or more healthy lifestyle in general. However, adjustment for socioeconomic and lifestyle factors, such as maternal education, maternal smoking during pregnancy, smoking in the home and breast feeding, 
did not materially alter the results. Neither did we find consistent effects of increased long-term intake of vegetables and fish, other foods that may reflect a healthy diet or lifestyle. However, since both socioeconomic status and a healthy lifestyle are complex concepts, residual confounding cannot be ruled out.

In 1997, BLACK and SHARPE [2] proposed that changes in dietary fat intake during the last few decades may have contributed to the increase in asthma and atopic disease in children. They argued that the decrease in intake of $n-3$ polyunsaturated fatty acids (PUFAs) from fish and saturated fats from butter and lard, and the increased intake of n-6 PUFAs from margarine and vegetable oils has led to an increased ratio of n-6 to n-3 fatty acid intake. This can result in increased production of arachidonic acid and prostaglandin $E_{2}$ with a consequent increase in the likelihood of atopic T-helper (Th) cell type 2 sensitisation, asthma and atopic disease [2]. Several studies have reported beneficial associations of dietary fish intake $[5,6$, $8,22,23,27-29]$ or harmful associations of margarine and vegetable oil intake $[4,9,23,27,29]$ with asthma and atopic disease in children, whereas other studies did not find effects of n-3 PUFAs [4, 7, 20] or n-6-rich foods [18]. In the present study, increased fish consumption at early age appeared to be protective for sensitisation to inhaled allergens and BHR, but seemed to increase the risk of sensitisation to food allergens at 8 yrs of age. We did not find any consistent effects for increased margarine consumption. The lack of clear findings for increased fish and margarine consumption might be due to the fact that the food frequency questionnaires (FFQs) were not detailed enough to estimate the intake of n-3 or n-6 fatty acids, or the ratio of these two. The effect mechanisms of dietary PUFA intake on inflammatory mediators and Th-cell differentiation are very complex, which makes it more likely that epidemiological studies produce conflicting results [30]. An earlier study of the same cohort has reported beneficial associations of daily consumption of full cream milk and butter at two years of age on wheeze and asthma at three years of age [7]. The present study shows that this association did not persist to 8 years of age.

The overall results of this study did not indicate strong differences between consumption at early or later age. The hypothesis that nutrients that exert effects on airway development or Th-cell differentiation are especially important during early life [30] could not be confirmed in this study. Probably, the time window of exposure relevant for effects on the developing airways and immune system is even earlier in life, before 2 yrs of age or during pregnancy. Previous analyses in this cohort on the longitudinal effects of maternal fruit consumption during pregnancy revealed an overall beneficial effect on wheeze. However, the association lost statistical significance after adjustment for potentially confounding factors [31].

Weaknesses of our study design were the lack of data on consumption of specific types of fruit, vegetables and fish. To understand more of the biological mechanisms underlying the associations between nutrition and asthma or allergy, more information about the intake of specific foods and nutrients is needed. If the effects observed in this study are caused by specific nutrients, estimating the intake of total food groups may have attenuated the effects on the asthma and allergy outcomes. Furthermore, the use of self-reported dietary data could have led to misclassification of dietary exposure. However, it is unlikely that this has happened nonrandomly. The FFQs were not validated in our study population. However, FFQ data are widely used in epidemiological studies, and provide reasonably valid and reproducible estimates to rank individuals according to food group [32]. We have chosen a limited number of foods and food groups based on reported associations with asthma or atopy in previous studies. However, analyses of associations between several food groups and several outcomes could have led to chance findings due to multiple testing. Incidental associations for dietary consumption of a certain food and only one of the investigated outcomes must therefore be interpreted with caution.

Our study population contained an intervention arm to investigate the effect of mite allergen avoidance by means of mite-impermeable mattress and pillow covers. This intervention arm only contained children born to atopic mothers, while in the natural history arm of the study, children born to nonatopic mothers were overrepresented. However, the proportion of atopic mothers in the total baseline study population was similar to the proportion of atopic mothers in the screened population $(\sim 30 \%)$. Furthermore, the intervention was shown to have no clinical effects at 1, 2, 4 [33], and $8 \mathrm{yrs}$ of age (data not shown), so we do not expect any consequences regarding the generalisability of the present results. Nevertheless, all analyses were adjusted for study arm and we checked for effect modification by maternal atopy, which was not the case. Online supplementary tables E2a-c show results excluding the intervention study population. These results were similar to those in the total study population, although some associations lost statistical significance due to the loss of power.

The strength of our study was the use of longitudinal dietary data. Dietary exposure during childhood changes over time, which might change associations with symptoms of asthma or atopy as well. Longitudinal dietary data can be used to investigate differences of consumption at early or later age, providing more evidence on possible mechanisms of effects.

In conclusion, the results of this study indicate no consistent effects of increased early or late consumption, or increased long-term intake of certain foods or food groups on asthma and allergy outcomes in 8-yr-old children, with a possible exception for fruit. Future prospective studies should use more detailed dietary data to better assess nutrient or food specific effects.

\section{SUPPORT STATEMENT}

The Prevention and Incidence of Asthma and Mite Allergy (PIAMA) study was financially supported by: the Netherlands Organisation for Health Research and Development; the Netherlands Organisation for Scientific Research; the Netherlands Asthma Fund; the Netherlands Ministry of Spatial Planning, Housing, and the Environment; the Netherlands Ministry of Health, Welfare and Sport; and the European Union.

\section{STATEMENT OF INTEREST}

Statements of interest for D.S. Postma and J.C. de Jongste can be found at www.erj.ersjournals.com/site/misc/statements.xhtml 


\section{ACKNOWLEDGEMENTS}

The authors gratefully acknowledge the participants in the PIAMA birth cohort study and all coworkers who helped conducting the medical examinations, field work and data management.

\section{REFERENCES}

1 Seaton A, Godden DJ, Brown K. Increase in asthma: a more toxic environment or a more susceptible population? Thorax 1994; 49: 171-174.

2 Black PN, Sharpe S. Dietary fat and asthma: is there a connection? Eur Respir I 1997; 10: 6-12.

3 Forastiere F, Pistelli R, Sestini P, et al. Consumption of fresh fruit rich in vitamin $\mathrm{C}$ and wheezing symptoms in children. Thorax 2000; 55: 283-288.

4 Farchi S, Forastiere F, Agabiti N, et al. Dietary factors associated with wheezing and allergic rhinitis in children. Eur Respir J 2003; 22: 772-780.

5 Antova T, Pattenden S, Nikiforov B, et al. Nutrition and respiratory health in children in six Central and Eastern European countries. Thorax 2003; 58: 231-236.

6 Hodge L, Salome C, Peat J, et al. Consumption of oily fish and childhood asthma risk. Med J Aust 1996; 164: 137-140.

7 Wijga AH, Smit HA, Kerkhof M, et al. Association of consumption of products containing milk fat with reduced asthma risk in preschool children: the PIAMA birth cohort study. Thorax 2003; 58: 567-572.

8 Tabak C, Wijga AH, de Meer G, et al. Diet and asthma in Dutch school children (ISAAC-2). Thorax 2006; 61: 1048-1053.

9 Bolte G, Frye C, Hoelscher B, et al. Margarine consumption and allergy in children. Am J Respir Crit Care Med 2001; 163: 277-279.

10 Pistelli R, Forastiere F, Corbo GM, et al. Respiratory symptoms and bronchial responsiveness are related to dietary salt intake and urinary potassium excretion in male children. Eur Respir J 1993; 6: 517-522.

11 Devereux G. Early life events in asthma - diet. Pediatr Pulmonol 2007; 42: 663-673.

12 Brunekreef B, Smit H, de Jongste J, et al. The prevention and incidence of asthma and mite allergy (PIAMA) birth cohort study: design and first results. Pediatr Allergy Immunol 2002; 13: Suppl. 15, 55-60.

13 Lakwijk N, van Strien RT, Doekes G, et al. Validation of a screening questionnaire for atopy with serum IgE tests in a population of pregnant Dutch women. Clin Exp Allergy 1998; 28: 454-458.

14 The International Study of Asthma and Allergies in Childhood (ISAAC) Steering Committee. Worldwide variations in the prevalence of symptoms of asthma, allergic rhinoconjunctivitis, and atopic eczema: ISAAC. Lancet 1998; 351: 1225-1232.

15 Cole TJ, Bellizzi MC, Flegal KM, et al. Establishing a standard definition for child overweight and obesity worldwide: international survey. BMJ 2000; 320: 1240-1243.
16 Cook DG, Carey IM, Whincup PH, et al. Effect of fresh fruit consumption on lung function and wheeze in children. Thorax 1997; 52: 628-633.

17 Wong GWK, Ko FWS, Hui DSC, et al. Factors associated with difference in prevalence of asthma in children from three cities in China: multicentre epidemiological survey. BMJ 2004; 329: 486.

18 Garcia-Marcos L, Canflanca IM, Garrido JB, et al. Relationship of asthma and rhinoconjunctivitis with obesity, exercise and Mediterranean Diet in Spanish schoolchildren. Thorax 2007; 62: 503-508.

19 Gilliland FD, Berhane KT, Li Y, et al. Children' lung function and antoxidant vitamin, fruit, juice, and vegetable intake. Am J Epidemiol 2003; 158: 576-584.

20 Hijazi N, Abalkhail B, Seaton A. Diet and childhood asthma in a society in transition: a study in urban and rural Saudi Arabia. Thorax 2000; 55: 775-779.

21 Huang SL, Lin KC, Pan WH. Dietary factors associated with physician-diagnosed asthma and allergic rhinitis in teenagers: analyses of the first nutrition and health survey in Taiwan. Clin Exp Allergy 2001; 31: 259-264.

22 Chatzi L, Torrent M, Romieu I, et al. Diet, wheeze, and atopy in school children in Menorca, Spain. Pediatr Allergy Immunol 2007; 18: $480-485$.

23 Chatzi L, Apostolaki G, Bibaki I, et al. Protective effects of fruits, vegetables, and the Mediterranean diet on asthma and allergies among children in Crete. Thorax 2007; 62: 677-683.

24 Hatch GE. Asthma, inhaled oxidants, and dietary antioxidants. Am J Clin Nutr 1995; 61: 625S-630S.

25 Tricon S, Willers S, Smit HA, et al. Nutrition and allergic disease. Clin Exp Allergy Rev 2006; 6: 117-188.

26 Fernandez-Rivas M, Benito C, Gonzalez-Manzebo E, et al. Allergies to fruit and vegetables. Pediatr Allergy Immunol 2008; 19: 675-681.

27 Dunder T, Kuikka L, Turtinen J, et al. Diet, serum fatty acids, and atopic diseases in childhood. Allergy 2001; 56: 425-428.

28 Andreasyan K, Ponsonby AL, Dwyer T, et al. A differing pattern of association between dietary fish and allergen-specific subgroups of atopy. Allergy 2005; 60: 671-677.

29 Kim JL, Elfman L, Mi Y, et al. Current asthma and respiratory symptoms among pupils in relation to dietary factors and allergens in the school environment. Indoor Air 2005; 15: 170-182.

30 Devereux $G$, Seaton A. Diet as a risk factor for atopy and asthma. I Allergy Clin Immunol 2005; 115: 1109-1117.

31 Willers SM, Wijga AH, Brunekreef B, et al. Maternal food consumption during pregnancy and the longitudinal development of childhood asthma. Am J Respir Crit Care Med 2008; 178: 124-131.

32 Biro G, Hulshof KFAM, Ovesen L, et al. Selection of methodology to assess food intake. Eur J Clin Nutr 2002; 56: 25-32.

33 Corver K, Kerkhof M, Brussee JE, et al. House dust mite allergen reduction and allergy at 4 yr: follow up of the PIAMA-study. Pediatr Allergy Immunol 2006; 17: 329-336. 